B. A. Bhayo, M. Vuorinen

\title{
INEQUALITIES FOR EIGENFUNCTIONS OF THE $P$-LAPLACIAN
}

\begin{abstract}
Motivated by the work of P. Lindqvist, we study eigenfunctions of the one-dimensional $p$-Laplace operator, the $\sin _{p}$ functions, and prove several inequalities for these and $p$-analogues of other trigonometric functions and their inverse functions. Similar inequalities are given also for the $p$-analogues of the hyperbolic functions and their inverses.
\end{abstract}

Key words: eigenfunctions of $p$-Laplacian, $\sin _{p}$, generalized trigonometric function.

2010 Mathematical Subject Classification: 33C99, 33B99.

\section{$\S 1$. Introduction}

In a highly cited paper P. Lindqvist [1] studied generalized trigonometric functions depending on a parameter $p>1$ which for the case $p=2$ reduce to the familiar functions. Numerous other authors, see e. g. [2], [3, 4], [5], [6], [7] and the bibliographies of these papers, have extended this work in various directions including the study of generalized hyperbolic functions and their inverses. Our goal here to study these $p$-trigonometric and $p$-hyperbolic functions and to prove several inequalities for them.

For the statement of some of our main results we introduce some notation and terminology for classical special functions, such as the classical gamma function $\Gamma(x)$, the psi function $\psi(x)$ and the beta function $B(x, y)$. For $\operatorname{Re} x>0, \operatorname{Re} y>0$, these functions are defined by

$$
\Gamma(x)=\int_{0}^{\infty} e^{-t} t^{x-1} d t, \psi(x)=\frac{\Gamma^{\prime}(x)}{\Gamma(x)}, B(x, y)=\frac{\Gamma(x) \Gamma(y)}{\Gamma(x+y)} .
$$

(C) Bhayo B. A., Vuorinen M., 2013 
Given complex numbers $a, b$ and $c$ with $c \neq 0,-1,-2, \ldots$, the Gaussian hypergeometric function is the analytic continuation to the slit place $\mathbf{C} \backslash[1, \infty)$ of the series

$$
F(a, b ; c ; z)={ }_{2} F_{1}(a, b ; c ; z)=\sum_{n=0}^{\infty} \frac{(a, n)(b, n)}{(c, n)} \frac{z^{n}}{n !}, \quad|z|<1 .
$$

Here $(a, 0)=1$ for $a \neq 0$, and $(a, n)$ is the shifted factorial function or the Appell symbol

$$
(a, n)=a(a+1)(a+2) \cdots(a+n-1)
$$

for $n \in \mathbf{N} \backslash\{0\}$, where $\mathbf{N}=\{0,1,2, \ldots\}$. The hypergeometric function has numerous special functions as its special or limiting cases, see [8].

We start by discussing eigenfunctions of the so-called one-dimensional $p$-Laplacian $\Delta_{p}$ on $(0,1), p \in(1, \infty)$. The eigenvalue problem [5]

$$
-\Delta_{p} u=-\left(\left|u^{\prime}\right|^{p-2} u^{\prime}\right)^{\prime}=\lambda|u|^{p-2} u, u(0)=u(1)=0,
$$

has eigenvalues

$$
\lambda_{n}=(p-1)\left(n \pi_{p}\right)^{p}
$$

and eigenfunctions

$$
\sin _{p}\left(n \pi_{p} t\right), n \in \mathbb{N},
$$

where $\sin _{p}$ is the inverse function of $\arcsin _{p}$, which is defined below and (cf. [7])

$$
\pi_{p}=\frac{2}{p} \int_{0}^{1}(1-s)^{-1 / p} s^{1 / p-1} d s=\frac{2}{p} B\left(1-\frac{1}{p}, \frac{1}{p}\right)=\frac{2 \pi}{p \sin (\pi / p)} .
$$

Motivated by P. Lindqvist's work, P. J. Bushell and D. E. Edmunds [9] found recently many new results for these generalized trigonometric functions. Some authors also considered various other $p$-analogues of trigonometric and hyperbolic functions and their inverses. In particular, they considered the following homeomorphisms

$$
\begin{gathered}
\sin _{p}:\left(0, a_{p}\right) \rightarrow I, \cos _{p}:\left(0, a_{p}\right) \rightarrow I, \tan _{p}:\left(0, b_{p}\right) \rightarrow I, \\
\sinh _{p}:\left(0, c_{p}\right) \rightarrow I, \tanh _{p}:(0, \infty) \rightarrow I,
\end{gathered}
$$


where $I=(0,1)$ and

$$
\begin{gathered}
a_{p}=\frac{\pi_{p}}{2}, b_{p}=\frac{1}{2 p}\left(\psi\left(\frac{1+p}{2 p}\right)-\psi\left(\frac{1}{2 p}\right)\right)=2^{-1 / p} F\left(\frac{1}{p}, \frac{1}{p} ; 1+\frac{1}{p} ; \frac{1}{2}\right), \\
c_{p}=\left(\frac{1}{2}\right)^{1 / p} F\left(1, \frac{1}{p} ; 1+\frac{1}{p} ; \frac{1}{2}\right) .
\end{gathered}
$$

For $x \in I$, their inverse functions are defined as

$$
\begin{aligned}
& \arcsin _{p} x=\int_{0}^{x}\left(1-t^{p}\right)^{-1 / p} d t=x F\left(\frac{1}{p}, \frac{1}{p} ; 1+\frac{1}{p} ; x^{p}\right)= \\
& =x\left(1-x^{p}\right)^{(p-1) / p} F\left(1,1 ; 1+\frac{1}{p} ; x^{p}\right), \\
& \arctan _{p} x=\int_{0}^{x}\left(1+t^{p}\right)^{-1} d t=x F\left(1, \frac{1}{p} ; 1+\frac{1}{p} ;-x^{p}\right), \\
& \operatorname{arsinh}_{p} x=\int_{0}^{x}\left(1+t^{p}\right)^{-1 / p} d t=x F\left(\frac{1}{p}, \frac{1}{p} ; 1+\frac{1}{p} ;-x^{p}\right), \\
& \operatorname{artanh}_{p} x=\int_{0}^{x}\left(1-t^{p}\right)^{-1} d t=x F\left(1, \frac{1}{p} ; 1+\frac{1}{p} ; x^{p}\right),
\end{aligned}
$$

and by [9, Prop. 2.2] $\arccos _{p} x=\arcsin _{p}\left(\left(1-x^{p}\right)^{1 / p}\right)$. See also S. Takeuchi [10] for two-parameter generalizations. For the particular case $p=2$ one obtains the familiar elementary functions.

The paper is organized into sections as follows. Section 1, the introduction, contains the statements of our main results. In Section 2 we give some inequalities for the $p$-analogues of trigonometric and hyperbolic functions. Section 3 contains the proofs of our main results and some identities. Finally in Section 4 we give some functional inequalities for elementary functions and Section 5 contains two small tables with a few values of the function $\sin _{p}$ and related functions compiled with the Mathematica ${ }^{\circledR}$ software.

Some of the main results are the following theorems.

Theorem 1. For $p>1$ and $x \in(0,1)$, we have

1) $\left(1+\frac{x^{p}}{p(1+p)}\right) x<\arcsin _{p} x<\frac{\pi_{p}}{2} x$, 
2) $\left(1+\frac{1-x^{p}}{p(1+p)}\right)\left(1-x^{p}\right)^{1 / p}<\arccos _{p} x<\frac{\pi_{p}}{2}\left(1-x^{p}\right)^{1 / p}$,

3) $\frac{\left(p(1+p)\left(1+x^{p}\right)+x^{p}\right) x}{p(1+p)\left(1+x^{p}\right)^{1+1 / p}}<\arctan _{p} x<2^{1 / p} b_{p}\left(\frac{x^{p}}{1+x^{p}}\right)^{1 / p}$.

Theorem 2. For $p>1$ and $x \in(0,1)$, we have

$$
\begin{aligned}
z\left(1+\frac{\log \left(1+x^{p}\right)}{1+p}\right) & <\operatorname{arsinh}_{p} x<z\left(1+\frac{1}{p} \log \left(1+x^{p}\right)\right), \\
z & =\left(\frac{x^{p}}{1+x^{p}}\right)^{1 / p}, \\
x\left(1-\frac{1}{1+p} \log \left(1-x^{p}\right)\right) & <\operatorname{artanh}_{p} x<x\left(1-\frac{1}{p} \log \left(1-x^{p}\right)\right) .
\end{aligned}
$$

The next result provides several families of inequalities for elementary functions.

Theorem 3. For $x>0$ and $z=\pi x / 2$, the function $g(p)=f\left(z^{p}\right)^{1 / p}$ is decreasing in $p \in(0, \infty)$, where

$$
f(z) \in\{\arcsin (2 z / \pi), \operatorname{arcosh}(z), \operatorname{artanh}(2 z / \pi)\} .
$$

Acknowledgments. The first author is indebted to the Graduate School of Mathematical Analysis and its Applications for support. The second author was, in part supported by the Academy of Finland, Project 2600066611. Both authors wish to acknowledge the expert help of Dr. H. Ruskeepää in the use of the Mathematica ${ }^{\circledR}$ software [11] and Prof. P. Hästö for providing simplified versions of some of our proofs. Authors thank to anonymous referee for his valuable comments.

\section{$\S 2$. Preliminaries and definitions}

For convenience, we use the notation $\mathbb{R}_{+}=(0, \infty)$.

Lemma 1. [13, Thm 2.1] Let $f: \mathbb{R}_{+} \rightarrow \mathbb{R}_{+}$be a differentiable, logconvex function and let $a \geq 1$. Then $g(x)=(f(x))^{a} / f(a x)$ decreases on its domain. In particular, if $0 \leq x \leq y$, then the following inequalities

$$
\frac{(f(y))^{a}}{f(a y)} \leq \frac{(f(x))^{a}}{f(a x)} \leq(f(0))^{a-1}
$$


hold true. If $0<a \leq 1$, then the function $g$ is an increasing function on $\mathbb{R}_{+}$and inequalities are reversed.

For easy reference we recall the following identity $[8,15.3 .5]$

$$
F(a, b ; c ; z)=(1-z)^{-b} F(b, c-a ; c ;-z /(1-z)) .
$$

For the following lemma see [15, Thms 1.19(10), 1.52(1), Lems, 1.33, $1.35]$.

\section{Lemma 2.}

1) For $a, b, c>0, c<a+b$, and $|x|<1$,

$$
F(a, b ; c ; x)=(1-x)^{c-a-b} F(c-a, c-b ; c ; x) .
$$

2) For $a, x \in(0,1)$, and $b, c \in(0, \infty)$

$$
F(-a, b ; c ; x)<1-\frac{a b}{c} x .
$$

3) For $a, x \in(0,1)$, and $b, c \in(0, \infty)$

$$
F(a, b ; c ; x)+F(-a, b ; c ; x)>2 .
$$

4) Let $a, b, c \in(0, \infty)$ and $c>a+b$. Then for $x \in[0,1]$,

$$
F(a, b ; c ; x) \leq \frac{\Gamma(c) \Gamma(c-a-b)}{\Gamma(c-a) \Gamma(c-b)} .
$$

5) For $a, b>0$, the following function

$$
f(x)=\frac{F(a, b ; a+b ; x)-1}{\log (1 /(1-x))}
$$

is strictly increasing from $(0,1)$ onto $(a b /(a+b), 1 / B(a, b))$.

Lemma 3. For $p>1$ and $x \in(0,1)$, the functions

$$
\left(\arcsin _{p}\left(x^{k}\right)\right)^{1 / k} \quad \text { and } \quad\left(\operatorname{artanh}_{p}\left(x^{k}\right)\right)^{1 / k}
$$

are decreasing in $k \in(0, \infty)$, also

$$
\left(\arctan _{p}\left(x^{k}\right)\right)^{1 / k} \quad \text { and } \quad\left(\operatorname{arsinh}_{p}\left(x^{k}\right)\right)^{1 / k}
$$

are increasing in $k \in(0, \infty)$. 
In particular, for $k \geq 1$

$$
\begin{aligned}
& \sqrt[k]{\arcsin _{p}\left(x^{k}\right)} \leq \arcsin _{p}(x) \leq\left(\arcsin _{p} \sqrt[k]{x}\right)^{k} \\
& \sqrt[k]{\operatorname{artanh}_{p}\left(x^{k}\right)} \leq \operatorname{artanh}_{p}(x) \leq\left(\operatorname{artanh}_{p} \sqrt[k]{x}\right)^{k} \\
& \left(\operatorname{arsinh}_{p} \sqrt[k]{x}\right)^{k} \leq \operatorname{arsinh}_{p}(x) \leq \sqrt[k]{\operatorname{arsinh}_{p}\left(x^{k}\right)} \\
& \left(\arctan _{p} \sqrt[k]{x}\right)^{k} \leq \arctan _{p}(x) \leq \sqrt[k]{\arctan _{p}\left(x^{k}\right)}
\end{aligned}
$$

Let

$$
f(k):=\left(E\left(x^{k}\right)\right)^{1 / k}, E(x):=\int_{0}^{x} g(t) d t, E=E\left(x^{k}\right) .
$$

We get

$$
\begin{gathered}
f^{\prime}=-E^{1 / k} \log E \frac{1}{k^{2}}+\frac{1}{k} E^{1 / k-1} E^{\prime} x^{k} \log x= \\
=\frac{E^{1 / k}}{k^{2}}\left(-\log \frac{E}{x^{k}}-\left(x^{k} \frac{E^{\prime}}{E}-1\right) \log \frac{1}{x^{k}}\right) .
\end{gathered}
$$

If $g \geq 1$, then

$$
\frac{E}{x^{k}}=\frac{1}{x^{k}} \int_{0}^{x^{k}} g(t) d t \geq 1
$$

If $g$ is increasing, then

$$
E^{\prime}-\frac{E}{x^{k}}=g\left(x^{k}\right)-\frac{1}{x^{k}} \int_{0}^{x^{k}} g(t) d t \geq 0,
$$

so that $x^{k} \frac{E^{\prime}}{E}-1 \geq 0$. Thus $f^{\prime} \leq 0$ under these assumptions.

For $\arcsin _{p}$ and $\operatorname{artanh}_{p}, g$ is $\left(1-t^{p}\right)^{-1 / p}$ and $\left(1-t^{p}\right)^{-1}$, so the conditions are clearly satisfied. Additionally, we see that for $\operatorname{arsinh}_{p}$ and $\arctan _{p}$ the conditions $g \leq 1$ and $g$ is decreasing and this conclude that $f^{\prime} \geq 0$. This completes the proof.

Theorem 4. For $p>1$ and $r, s \in(0,1)$, the following inequalities hold

1) $\arcsin _{p}(r s) \leq \sqrt{\arcsin _{p}\left(r^{2}\right) \arcsin _{p}\left(s^{2}\right)} \leq \arcsin _{p}(r) \arcsin _{p}(s)$, $r, s \in(0,1)$, 
2) $\operatorname{artanh}_{p}(r s) \leq \sqrt{\operatorname{artanh}_{p}\left(r^{2}\right) \operatorname{artanh}_{p}\left(s^{2}\right)} \leq \operatorname{artanh}_{p}(r) \operatorname{artanh}_{p}(s)$, $r, s \in(0,1)$,

3) $\operatorname{arsinh}_{p}\left(r^{2}\right) \operatorname{arsinh}_{p}\left(s^{2}\right) \leq \sqrt{\operatorname{arsinh}_{p}\left(r^{2}\right) \operatorname{arsinh}_{p}\left(s^{2}\right)} \leq \operatorname{arsinh}_{p}(r s)$,

4) $\operatorname{artanh}_{p}(r) \operatorname{artanh}_{p}(s) \leq \sqrt{\operatorname{artanh}_{p}\left(r^{2}\right) \operatorname{artanh}_{p}\left(s^{2}\right)} \leq \operatorname{artanh}_{p}(r s)$.

Let $h(x):=\log f\left(e^{x}\right)$. Then $h$ is convex (in the $C^{2}$ case) when $h^{\prime \prime} \geq 0$, i. e. if

$$
\frac{f}{y}\left(f^{\prime}+y f^{\prime \prime}\right) \geq\left(f^{\prime}\right)^{2}
$$

where $y=e^{x}$ and the function is evaluated at $y$. If $f^{\prime \prime} \geq 0$, then

$$
\frac{f}{y} \geq f^{\prime}(0)
$$

so a sufficient condition for convexity is $f^{\prime}(0)\left(f^{\prime}+y f^{\prime \prime}\right) \geq\left(f^{\prime}\right)^{2}$. If $f^{\prime \prime} \leq 0$, the reverse holds, so a sufficient condition for concavity is $f^{\prime}(0)\left(f^{\prime}+y f^{\prime \prime}\right) \leq$ $\leq\left(f^{\prime}\right)^{2}$. Suppose

$$
f(x):=\int_{0}^{x} g(t) d t .
$$

Then $f^{\prime}=g$ and $f^{\prime \prime}=g^{\prime}$. Then one easily checks that $h$ is convex in case $g$ is $\left(1-t^{p}\right)^{-1 / p}$ and $\left(1-t^{p}\right)^{-1}$, and concave for $g$ equal to $\left(1+t^{p}\right)^{-1 / p}$ and $\left(1+t^{p}\right)^{-1}$. Now proof follows easily from Lemma 3 .

Lemma 4. For $k, p>1$ and $r \geq s$, we have

$$
\begin{aligned}
& \left(\frac{\arcsin _{p}(s)}{\arcsin _{p}(r)}\right)^{k} \leq \frac{\arcsin _{p}\left(s^{k}\right)}{\arcsin \left(r^{k}\right)}, r, s \in(0,1), \\
& \left(\frac{\operatorname{artanh}_{p}(s)}{\operatorname{artanh}_{p}(r)}\right)^{k} \leq \frac{\operatorname{artanh}_{p}\left(s^{k}\right)}{\operatorname{artanh}_{p}\left(r^{k}\right)}, r, s \in(0,1), \\
& \frac{\operatorname{arsinh}_{p}\left(s^{k}\right)}{\operatorname{arsinh}_{p}\left(r^{k}\right)} \leq\left(\frac{\operatorname{arsinh}_{p}(s)}{\operatorname{arsinh}_{p}(r)}\right)^{k}, r, s \in(0,1) .
\end{aligned}
$$

For $x>0$, the following functions

$$
\begin{gathered}
u(x)=\arcsin _{p}\left(e^{-x}\right), \quad v(x)=\operatorname{artanh}_{p}\left(e^{-x}\right), \\
w_{1}(x)=1 / \operatorname{arsinh}_{p}\left(e^{-x}\right)
\end{gathered}
$$

are log-convex by the proof of Theorem 4 . Let $x<y, e^{-x}=r \geq s=e^{-y}$, now inequalities follow from Lemma 1. 
Lemma 5. [17, Thm 2, p.151] Let $J \subset \mathbb{R}$ be an open interval, and let $f: J \rightarrow \mathbb{R}$ be strictly monotonic function. Let $f^{-1}: f(J) \rightarrow J$ be the inverse to $f$ then

1) if $f$ is convex and increasing, then $f^{-1}$ is concave,

2) if $f$ is convex and decreasing, then $f^{-1}$ is convex,

3 ) if $f$ is concave and increasing, then $f^{-1}$ is convex,

4) if $f$ is concave and decreasing, then $f^{-1}$ is concave.

Lemma 6. For $k, p>1$ and $r \geq s$, we have

$$
\begin{aligned}
\left(\frac{\sin _{p}(r)}{\sin _{p}(s)}\right)^{k} & \leq \frac{\sin _{p}\left(r^{k}\right)}{\sin _{p}\left(s^{k}\right)}, r, s \in(0,1), \\
\left(\frac{\tanh _{p}(r)}{\tanh _{p}(s)}\right)^{k} & \leq \frac{\tanh _{p}\left(r^{k}\right)}{\tanh _{p}\left(s^{k}\right)}, r, s \in(0, \infty), \\
\left(\frac{\sinh _{p}(r)}{\sinh _{p}(s)}\right)^{k} & \geq \frac{\sinh _{p}\left(r^{k}\right)}{\sinh _{p}\left(s^{k}\right)}, r, s \in(0,1) .
\end{aligned}
$$

Inequalities reverse for $k \in(0,1)$.

It is clear from the proof of Theorem 4 that the functions

$$
\begin{gathered}
f(x)=\log \left(\arcsin _{\mathrm{p}}\left(e^{-x}\right)\right), g(x)=\log \left(\operatorname{artanh}_{\mathrm{p}}\left(e^{-x}\right)\right), \\
h(x)=\log \left(1 / \operatorname{arsinh}_{\mathrm{p}}\left(e^{x}\right)\right)
\end{gathered}
$$

are convex and decreasing, then Lemma 5(2) implies that

$$
\begin{gathered}
f^{-1}(y)=\log \left(1 / \sin _{p}\left(e^{y}\right)\right), g^{-1}(y)=\log \left(1 / \tanh _{p}\left(e^{y}\right)\right), \\
h^{-1}(y)=\log \left(\sinh _{p}\left(e^{-y}\right)\right),
\end{gathered}
$$

are convex, now the result follows from Lemma 1.

Theorem 5. For $p>1$, the following inequalities hold

$$
\begin{aligned}
& \text { 1) } \sqrt{\sin _{p}\left(r^{2}\right) \sin _{p}\left(s^{2}\right)} \leq \sin _{p}(r s), r, s \in\left(0, \pi_{p} / 2\right), \\
& \text { 2) } \sqrt{\tanh _{p}\left(r^{2}\right) \tanh _{p}\left(s^{2}\right)} \leq \tanh _{p}(r s), r, s \in(0, \infty), \\
& \text { 3) } \sinh _{p}(r s) \leq \sqrt{\sinh _{p}\left(r^{2}\right) \sinh _{p}\left(s^{2}\right)}, \quad r, s \in(0, \infty)
\end{aligned}
$$


Let $f(z)=\log \left(\arcsin _{p}\left(e^{-z}\right)\right), z>0$. Then

$$
f^{\prime}(z)=-\left(1-e^{-p z}\right)^{-1 / p} / F\left(1 / p, 1 / p ; 1+1 / p ; e^{-p z}\right)<0,
$$

$f$ is decreasing and by the proof of Theorem $4 f$ is convex. By Lemma $5(2), f^{-1}(y)=\log \left(1 / \sin _{p}\left(e^{y}\right)\right)$ is convex. This implies that

$$
\log \left(\frac{1}{\sin _{p}\left(e^{x / 2} e^{y / 2}\right)}\right) \leq \frac{1}{2}\left(\log \left(\frac{1}{\sin _{p}\left(e^{x}\right)}\right)+\log \left(\frac{1}{\sin _{p}\left(e^{y}\right)}\right)\right),
$$

letting $r=e^{x / 2}$ and $s=e^{y / 2}$, we get the first inequality.

For $(2)$, let $g(z)=\log \left(\operatorname{artanh}_{p}\left(e^{-z}\right)\right), z>0$ and

$$
g^{\prime}(z)=-1 /\left(\left(1-e^{-p z}\right) F\left(1,1 / p ; 1+1 / p ; e^{-p z}\right)\right)<0,
$$

hence $g$ is decreasing and by Theorem $4 g$ is convex. Then $g^{-1}(y)=$ $=\log \left(1 / \operatorname{artanh}_{p}\left(e^{y}\right)\right)$ is convex by Lemma $5(2)$, and (2) follows. Finally, let $h_{1}(z)=\log \left(1 / \operatorname{arsinh}_{p}\left(e^{z}\right)\right)$ and

$$
h_{1}^{\prime}(z)=-1 / F\left(1,1 / p ; 1+1 / p ; \frac{e^{p z}}{1+e^{p z}}\right)<0 .
$$

Then $h_{1}^{-1}(y)=\log \left(\sinh _{p}\left(e^{-y}\right)\right)$ is decreasing and convex by Lemma $5(2)$. This implies that

$$
\log \left(1 / \sinh _{p}\left(e^{-x / 2} e^{-y / 2}\right)\right) \leq\left(\log \left(1 / \sinh _{p}\left(e^{-x}\right)\right)+\log \left(1 / \sinh _{p}\left(e^{-y}\right)\right)\right) / 2,
$$

and (3) holds for $r, s \in(0, \infty)$. Again $h_{2}(z)=\log \left(1 / \operatorname{arsinh}_{p}\left(e^{-z}\right)\right)$ and

$$
h_{2}^{\prime}(z)=\left(F\left(1,1 / p ; 1+1 / p ; 1 /\left(1+e^{p z}\right)\right)\right)^{-1}>0
$$

similarly proof follows from Lemma 5(2), this completes the proof of (3).

Lemma 7. For $p>1$, the following relations hold

1) $\sqrt{\sin _{p}(r) \sin _{p}(s)} \leq \sin _{p}((r+s) / 2), r, s \in\left(0, \pi_{p} / 2\right)$,

2) $\sqrt{\sinh _{p}(r) \sinh _{p}(s)} \leq \sinh _{p}((r+s) / 2), r, s \in(0, \infty)$.

The proof follows easily from Lemma 5 and $2 \sqrt{r s} \leq r+s$ since the functions are increasing. 
Lemma 8. For $p>1$, the following inequalities hold

1) $\sin _{p}(r+s) / 2 \leq\left(\sin _{p}(r)+\sin _{p}(s)\right) / 2, r, s \in\left(0, \pi_{p} / 2\right)$,

2) $\tanh _{p}(r+s) / 2 \leq\left(\tanh _{p}(r)+\tanh _{p}(s)\right) 2, r, s \in\left(0, b_{p} / 2\right)$,

3) $\tan _{p}(r+s) / 2 \geq\left(\tan _{p}(r)+\tan _{p}(s)\right) 2, r, s \in\left(0, b_{p} / 2\right)$,

4) $\sinh _{p}(r+s) / 2 \geq\left(\sinh _{p}(r)+\sinh _{p}(s)\right) 2, r, s \in(0, \infty)$.

Let $f(x)=\arcsin _{p}(x), x \in\left(0, a_{p}\right)$. We get

$$
f^{\prime}(x)=\left(1-x^{p}\right)^{-1 / p},
$$

which is increasing, hence $f$ is convex. Clearly, $f$ is increasing. Therefore

$$
f_{1}=f^{-1}(y)=\sin _{p}(y)
$$

is concave by Lemma $5(1)$. This implies that $f_{1}^{\prime}$ is decreasing. Clearly $f_{1}(0)=0$, and by [15, Thm 1.25], $f_{1}(y) / y$ is decreasing. Now it follows from [15, Lem 1.24] that

$$
f_{1}(r+s) \leq f_{1}(r)+f_{1}(s)
$$

and (1) follows. The proofs of the remaining claims follow similarly.

\section{$\S 3$. Proof of main results}

In this section we give the proof of our main results and few more relations.

Proof of Theorem 1. By Lemma 2(3), (2) we get

$$
2-\left(1-\frac{x^{p}}{p(1+p)}\right)<F\left(\frac{1}{p}, \frac{1}{p} ; 1+\frac{1}{p} ; x^{p}\right)
$$

and the first inequality of part one holds. For the second one we get

$$
\begin{gathered}
\arcsin _{p} x=x F\left(\frac{1}{p}, \frac{1}{p} ; 1+\frac{1}{p} ; x^{p}\right)< \\
<\frac{x \Gamma(1+1 / p) \Gamma(1+1 / p-1 / p-1 / p)}{\Gamma(1+1 / p-1 / p) \Gamma(1+1 / p-1 / p)}= \\
=x \Gamma\left(1+\frac{1}{p}\right) \Gamma\left(1-\frac{1}{p}\right)=x \frac{1}{p} B\left(1-\frac{1}{p}, \frac{1}{p}\right)=x \frac{\pi_{p}}{2}
\end{gathered}
$$


by Lemma 2(4). By [9, Prop. (2.11)], $\arccos _{p} x=\arcsin _{p}\left(\left(1-x^{p}\right)^{1 / p}\right)$, and (2) follows from (1). For (3), if we replace $b=1, c-a=1 / p, c=$ $=1+1 / p, x^{p}=z /(1-z)$ in $(3)$ then we get

$$
\begin{aligned}
\arctan _{p} x & =x F\left(1, \frac{1}{p} ; 1+\frac{1}{p} ;-x^{p}\right)= \\
& =\left(\frac{x}{1+x^{p}}\right) F\left(1,1 ; 1+\frac{1}{p} ; \frac{x^{p}}{1+x^{p}}\right)= \\
& =\left(\frac{x}{1+x^{p}}\right)\left(\frac{1}{1+x^{p}}\right)^{1 / p-1} F\left(\frac{1}{p}, \frac{1}{p} ; 1+\frac{1}{p} ; \frac{x^{p}}{1+x^{p}}\right)= \\
& =\left(\frac{x^{p}}{1+x^{p}}\right)^{1 / p} F\left(\frac{1}{p}, \frac{1}{p} ; 1+\frac{1}{p} ; \frac{x^{p}}{1+x^{p}}\right)< \\
& <2^{1 / p} b_{p}\left(\frac{x^{p}}{1+x^{p}}\right)^{1 / p},
\end{aligned}
$$

third identily and inequality follow from Lemma 2(1), (4). For the lower bound we get

$$
\begin{aligned}
\arctan _{p} x & >\left(\frac{x^{p}}{1+x^{p}}\right)^{1 / p}\left(2-F\left(\frac{1}{p}, \frac{1}{p} ; 1+\frac{1}{p} ; \frac{x^{p}}{1+x^{p}}\right)\right)> \\
& >\frac{\left(p(1+p)\left(1+x^{p}\right)+x^{p}\right) x}{p(1+p)\left(1+x^{p}\right)^{1+1 / p}}
\end{aligned}
$$

from Lemma 2(3),(2).

Proof of Theorem 2. For (1), we replace $b=1 / p, c-a=1 / p, c=$ $=1+1 / p$ and $x^{p}=z /(1-z)$ in (3) and see that

$$
\begin{aligned}
& \operatorname{arsinh}_{p} x=x F\left(\frac{1}{p}, \frac{1}{p} ; 1+\frac{1}{p} ;-x^{p}\right)= \\
& =\left(\frac{x^{p}}{1+x^{p}}\right)^{1 / p} F\left(1, \frac{1}{p} ; 1+\frac{1}{p} ; \frac{x^{p}}{1+x^{p}}\right) .
\end{aligned}
$$

Now we get

$$
\begin{gathered}
\frac{\log \left(1+x^{p}\right)}{1+p}\left(\frac{x^{p}}{1+x^{p}}\right)^{1 / p}< \\
<x F\left(\frac{1}{p}, \frac{1}{p} ; 1+\frac{1}{p} ;-x^{p}\right)<\left(1-\frac{1}{p} \log \left(1-\frac{x^{p}}{1+x^{p}}\right)\right)\left(\frac{x^{p}}{1+x^{p}}\right)^{1 / p}
\end{gathered}
$$

from Lemma $2(5)$ and observing that $B(1,1 / p)=p$, this implies (1). 
For (2) we get from Lemma 2(5)

$$
\frac{1}{1+p} \log \left(\frac{1}{1-x^{p}}\right)+1<F\left(1, \frac{1}{p} ; 1+\frac{1}{p} ; x^{p}\right)<\frac{1}{p} \log \left(\frac{1}{1-x^{p}}\right)+1
$$

which is equivalent to

$$
\begin{gathered}
x\left(1-\frac{1}{1+p} \log \left(1-x^{p}\right)\right)<x F\left(1, \frac{1}{p} ; 1+\frac{1}{p} ; x^{p}\right)< \\
<x\left(1-\frac{1}{p} \log \left(1-x^{p}\right)\right),
\end{gathered}
$$

and the result follows.

Remark. For the particular case $p=2$. Zhu [18] has proved for $x>0$

$$
\frac{6 \sqrt{2}\left(\sqrt{1+x^{2}}-1\right)^{1 / 2}}{4+\sqrt{2}\left(\sqrt{1+x^{2}}+1\right)^{1 / 2}}<\operatorname{arsinh}(x) .
$$

When $p=2$, our bound in Theorem 2(1) differs from this bound roughly 0.01 when $x \in(0,1)$.

Lemma 9. For $p>1$ and $x \in(0,1)$, the following inequalities hold:

1) $\arctan _{p}(x)<\operatorname{arsinh}_{p}(x)<\arcsin _{p}(x)<\operatorname{artanh}_{p}(x)$,

2) $\tanh _{p}(z)<\sin _{p}(z)<\sinh _{p}(z)<\tan _{p}(z)$,

the first and the second inequalities hold for $z \in\left(0, \pi_{p} / 2\right)$, and the third one holds for $z \in\left(0, b_{p}\right)$.

From the definition of the $p$-analogues functions we get (1), and (2) follows from (1).

Lemma 10. For $p>1$, we have

$$
\frac{6 p^{2}}{3 p^{2}-2} \leq \pi_{p} \leq \frac{12 p^{2}}{6 p^{2}-\pi^{2}}, \pi_{p}=\frac{2 \pi}{p \sin (\pi / p)}
$$

By [19, Thm 3.1] we get

$$
\frac{\pi}{p}\left(1-\frac{\pi^{2}}{6 p^{2}}\right) \leq \sin \left(\frac{\pi}{p}\right) \leq \frac{\pi}{p}\left(1-\frac{2}{3 p^{2}}\right),
$$

and the result follows easily. 
Lemma 11. For $a \in(0,1)$ and $k, r, s \in(1, \infty)$, the following inequalities hold

1) $\pi_{r s} \leq \sqrt{\pi_{r^{2}} \pi_{s^{2}}} \leq \sqrt{\pi_{r} \pi_{s}}$,

2) $\pi_{r^{a} s^{1-a}} \leq a \pi_{r}+(1-a) \pi_{s}$,

3) $\left(\frac{\pi_{s}}{\pi_{r}}\right)^{k} \leq \frac{\pi_{s^{k}}}{\pi_{r^{k}}}, r \leq s$.

Let $f(x)=\log \left(\pi_{e^{x}}\right), x>0$. We get

$$
f^{\prime \prime}(x)=e^{-2 x} \pi^{2}\left(\csc \left(e^{-x} \pi\right)\right)^{2}-e^{-x} \pi \cot \left(e^{-x} \pi\right),
$$

which is positive, because the function $g(y)=y^{2}(\csc (y))^{2}-y \cot (y)$ is positive. This implies that $f$ is convex. Hence

$$
\log \left(\pi_{e^{(x+y) / 2}}\right) \leq \frac{1}{2}\left(\log \left(\pi_{e^{x}}\right)+\log \left(\pi_{e^{y}}\right)\right)
$$

setting $r=e^{x / 2}$ and $r=e^{y / 2}$, we get the first inequality of (1), and the second one follows from the fact that $\pi_{p}$ is decreasing in $p \in(1, \infty)$. Now it is clear that $\pi_{e^{x}}$ is convex, and we get

$$
\pi_{e^{a x+(1-a) y}} \leq a \pi_{e^{x}}+(1-a) \pi_{e^{y}}
$$

and (2) follows easily. Let $0 \leq x \leq y$, then we get

$$
\frac{\left(\pi_{e^{y}}\right)^{k}}{\pi_{e^{k y}}} \leq \frac{\left(\pi_{e^{x}}\right)^{k}}{\pi_{e^{k x}}}
$$

from Lemma 1, and (3) follows if we set $r=e^{x}$ and $r=e^{y}$.

Lemma 12. For $p>1$ and $x \in(0,1)$, we have

$$
\begin{aligned}
& \arcsin _{p}\left(\frac{x}{\sqrt[p]{1+x^{p}}}\right)=\arctan _{p}(x), \\
& \arcsin _{p}(x)=\arctan _{p}\left(\frac{x}{\sqrt[p]{1-x^{p}}}\right), \\
& \arccos _{p}(x)=\arctan _{p}\left(\frac{\sqrt[p]{1-x^{p}}}{x}\right), \\
& \arccos _{p}\left(\frac{1}{\sqrt[p]{1+x^{p}}}\right)=\arctan _{p}(x) .
\end{aligned}
$$


We get

$$
\begin{aligned}
\arctan _{p}(x) & =x F\left(\frac{1}{p}, \frac{1}{p} ; 1+\frac{1}{p} ;-x^{p}\right)= \\
& =\frac{x}{1+x^{p}} F\left(1,1 ; 1+\frac{1}{p} ; \frac{x^{p}}{1+x^{p}}\right)= \\
& =\frac{x}{1+x^{p}}\left(\frac{1}{1+x^{p}}\right)^{1 / p-1} F\left(\frac{1}{p}, \frac{1}{p} ; 1+\frac{1}{p} ; \frac{x^{p}}{1+x^{p}}\right)= \\
& =\left(\frac{x}{1+x^{p}}\right)^{1 / p} F\left(\frac{1}{p}, \frac{1}{p} ; 1+\frac{1}{p} ;\left(\frac{x}{\left(1+x^{p}\right)^{1 / p}}\right)^{p}\right)= \\
& =\arcsin _{p}\left(\frac{x}{\sqrt[p]{1+x^{p}}}\right)
\end{aligned}
$$

by (3) and Lemma 2(1). Write $y=x / \sqrt[p]{1-x^{p}}$, and second follows from first one. For the third identity, we get

$$
\begin{aligned}
\arctan _{p}\left(\frac{\sqrt[p]{1-x^{p}}}{x}\right) & =x \sqrt[p]{1-x^{p}} F\left(\frac{1}{p}, \frac{1}{p} ; 1+\frac{1}{p} ;\left(1-x^{p}\right)\right)= \\
& =\arcsin _{p}\left(\left(1-x^{p}\right)^{1 / p}\right)=\arccos _{p}(x)
\end{aligned}
$$

by (3), Lemma 2(1) and [9, Prop. 2.2]. Similarly, the fourth identity follows from third one.

Conjecture 1. For a fixed $x \in(0,1)$, the functions

$$
\sin _{p}\left(\pi_{p} x / 2\right), \tan _{p}\left(\pi_{p} x / 2\right), \sinh _{p}\left(c_{p} x\right)
$$

are monotone in $p \in(1, \infty)$. For fixed $x>0, \tanh _{p}(x)$ is increasing in $p \in(1, \infty)$.

\section{$\S 4$. Some relations for elementary functions}

In this section we give several inequalities involving the elementary functions in form of Lemmas.

Lemma 13. For $x \in(0,1)$ and $z \in(0, \infty)$, the following functions

$$
f_{1}(k)=\sin \left(x^{k}\right)^{1 / k}, f_{2}(k)=\cos \left(x^{k}\right)^{1 / k}, f_{3}(k)=\tanh \left(z^{k}\right)^{1 / k},
$$

are increasing in $(0, \infty)$. 
We get

$$
f_{1}^{\prime}(k)=\left(x^{k} \cot \left(x^{k}\right) \log \left(x^{k}\right)-\log \left(\sin \left(x^{k}\right)\right)\right) \sin \left(x^{k}\right)^{1 / k} / k^{2},
$$

which is positive because

$$
h_{1}(y)=y \cot (y) \log (y)-\log (\sin (y)) \geq 0 .
$$

For $f_{2}$ we get

$$
f_{2}^{\prime}(k)=-\left(x^{k} \tan \left(x^{k}\right) \log \left(x^{k}\right)+\log \left(\cos \left(x^{k}\right)\right)\right) \cos \left(x^{k}\right)^{1 / k} / k^{2},
$$

which is positive because the function

$$
h_{2}(y)=y \tan (y) \log (y)+\log (\cos (y)) \leq 0 .
$$

For $f_{3}$ we get

$$
f_{3}^{\prime}(k)=\frac{\tanh \left(z^{k}\right)^{1 / k}}{k^{2}}\left(2 z^{k} \log \left(z^{k}\right) / \sinh \left(2 z^{k}\right)-\log \left(\tanh \left(z^{k}\right)\right)\right) .
$$

Let $h_{3}(y)=2 y \log (y) / \sinh (2 y)-\log (\tanh (y)), y=z^{k} \in(0, \infty)$. Clearly $h_{3}(y)>0$ for $y>1$. For $y \in(0,1)$ we see that $h_{3}(y)>0$ if

$$
\frac{2 y}{\sinh (2 y)} \frac{\log (y)}{\log (\tanh (y))} \leq 1
$$

which holds because $y>\tanh (y)$. In conclusion, $f_{3}^{\prime}(k)>0$ for all $z \in(0, \infty)$.

Lemma 14. The following inequalities hold

1) $\sqrt{\arccos \left(r^{2}\right) \arccos \left(s^{2}\right)}<\arccos (r s), r, s \in(0,1)$,

2) $\arctan (r) \arctan (s)<\sqrt{\arctan \left(r^{2}\right) \arctan \left(s^{2}\right)}<\arctan (r s)$, for $r, s \in(0,1)$,

3) $\sqrt{\operatorname{arcosh}\left(r^{2}\right) \operatorname{arcosh}\left(s^{2}\right)}<\operatorname{arcosh}(r s) ; r, s \in(1, \infty)$.

For (1) we let $f(x)=\log \left(\arccos \left(e^{-x}\right)\right), x>0$, and get

$$
f^{\prime \prime}(x)=-\frac{\sqrt{e^{2 x}-1}+e^{2 x} \arccos \left(e^{-x}\right)}{\left(e^{2 x}-1\right)^{3 / 2} \arccos ^{2}\left(e^{-x}\right)} \leq 0,
$$

hence $f$ is concave, and the inequality follows. 
For (2) we define $g(x)=\log \left(\arcsin \left(e^{-x}\right)\right), x>0$ and obtain

$$
g^{\prime \prime}(x)=\frac{e^{x}\left(\left(e^{2 x}-1\right) \tan ^{-1}\left(e^{-x}\right)-e^{x}\right)}{\left(e^{2 x}+1\right)^{2} \tan ^{-1}\left(e^{-x}\right)^{2}}<0,
$$

because $y<\tan \left(y /\left(1-y^{2}\right)\right)$ for $y \in(0,1)$, hence $g$ is concave. Therefore the first inequality of (2) follows and the second one follows from Lemma 13. Finally we define $h(x)=\log \left(\operatorname{arcosh}\left(e^{x}\right)\right), x>0$ and get

$$
h^{\prime \prime}(x)=-\frac{e^{x}\left(e^{x} \sqrt{e^{2 x}-1}-\operatorname{arcosh}\left(e^{x}\right)\right)}{\left(e^{2 x}-1\right)^{3 / 2} \operatorname{arcosh}^{2}\left(e^{x}\right)}<0 .
$$

This implies the proof of (3).

Lemma 15. For $r, s \in(0, \infty)$, we have

1) $\cosh (r s)<\sqrt{\cosh \left(r^{2}\right) \cosh \left(s^{2}\right)}<\cosh (r) \cosh (s)$, here second inequality holds for $r, s \in(0,1)$,

2) $\tanh (r) \tanh (s)<\sqrt{\tanh \left(r^{2}\right) \tanh \left(s^{2}\right)}<\sqrt{\tanh \left(r^{2} s^{2}\right)}$.

For $(1)$ we let $g_{1}(x)=\log \left(\cosh \left(e^{-x}\right)\right)$ and $g_{2}(x)=\log \left(\cosh \left(e^{x}\right)\right), x>0$, and we get

$$
\begin{gathered}
g_{1}^{\prime \prime}(x)=e^{-2 x}\left(1 /\left(\cosh ^{2}\left(e^{-x}\right)\right)+e^{x} \tanh \left(e^{-x}\right)\right)>0, \\
g_{2}^{\prime \prime}(x)=e^{x}\left(e^{x} /\left(\cosh ^{2}\left(e^{x}\right)\right)+\tanh \left(e^{x}\right)\right)>0,
\end{gathered}
$$

hence $g_{1}$ and $g_{2}$ are convex, and the first inequality of (1) holds, and its second inequality follows from Lemma 13. The firstinequality of (2) follows from Lemma 13. For the second one let $h_{1}(x)=\log \left(\tanh \left(e^{-x}\right)\right), x>0$ and get

$$
e^{-2 x}\left(-\operatorname{csch}^{2}\left(e^{-x}\right)+2 e^{x} \operatorname{csch}\left(2 e^{-x}\right)-\operatorname{sech}^{2}\left(e^{-x}\right)\right)
$$

which is negative, hence $h_{1}$ is concave. Again, let $h_{2}(x)=\log \left(\tanh \left(e^{x}\right)\right)$ and get

$$
-e^{x}\left(e^{x} \operatorname{csch}^{2}\left(e^{x}\right)-2 \operatorname{csch}\left(2 e^{x}\right)+e^{x} \operatorname{sech}^{2}\left(e^{x}\right)\right)<0 .
$$

This implies that $h_{2}$ is also concave, and the second inequality of (2) holds for $r, s \in(0, \infty)$. 
Lemma 16. For $y \in(0,1)$, we have

$$
\begin{gathered}
\frac{\pi}{2} y \cot \left(\frac{\pi y}{2}\right) \log y \leq \log \left(\sin \left(\frac{\pi y}{2}\right)\right), \\
y \operatorname{coth}(y) \log y \leq \log (\sinh (y)), \\
\log \left(\tan \left(\frac{\pi y}{2}\right)\right) \geq \frac{\pi}{2} y \log (y) \csc \left(\frac{\pi y}{2}\right) \sec \left(\frac{\pi y}{2}\right) .
\end{gathered}
$$

Let $f(y)=\frac{\pi}{2} y \cot \left(\frac{\pi y}{2}\right) \log y-\log \left(\sin \left(\frac{\pi y}{2}\right)\right)$. We get

$$
\begin{aligned}
f^{\prime}(y) & =\frac{\pi}{2} \cot \left(\frac{\pi y}{2}\right) \log y-\frac{1}{4} y \pi^{2} \csc ^{2}\left(\frac{\pi y}{2}\right) \log y= \\
& =\frac{\pi}{2} \log \left(y^{-1}\right)\left(\frac{\pi y}{2} \frac{1}{\sin ^{2}(\pi y / 2)}-\frac{\cos (\pi y / 2)}{\sin (\pi y / 2)}\right)= \\
& =\frac{\pi}{2} \frac{\log \left(y^{-1}\right)}{\sin ^{2}(\pi y / 2)}\left(\frac{\pi y}{2}-\sin \left(\frac{\pi y}{2}\right) \cos \left(\frac{\pi y}{2}\right)\right)= \\
& =\frac{\pi}{2} \frac{\log \left(y^{-1}\right)}{\sin ^{2}(\pi y / 2)}\left(\frac{\pi y}{2}-\frac{\sin (\pi y)}{2}\right) .
\end{aligned}
$$

This is positive because $x \geq \sin x$ for $x \in(0,2 \pi)$, and $f(1)=0$ and this completes the proof. Next, let

$$
g(y)=y \operatorname{coth}(y) \log y-\log (\sinh (y)) .
$$

We get

$$
g^{\prime}(y)=\frac{\log (1 / y)}{\sinh ^{2}(y)}(y-\sinh (y) \cosh (y)) \leq 0,
$$

because $\sinh x \geq x / \cosh x$ for $x>0$. Moreover, $g$ tends to zero when $y$ tends to zero and this implies the proof of (5). Next, let

$$
h(y)=\log \left(\tan \left(\frac{\pi y}{2}\right)\right)-\frac{\pi}{2} y \log (y) \csc \left(\frac{\pi y}{2}\right) \sec \left(\frac{\pi y}{2}\right) .
$$

We see that

$$
\begin{aligned}
h^{\prime}(y)= & -\frac{\pi^{2}}{4} y \log (y) \sec ^{2}\left(\frac{\pi y}{2}\right)+\frac{1}{4} \pi^{2} y \log (y) \csc ^{2}\left(\frac{\pi y}{2}\right)- \\
& -\frac{\pi}{2} \log (y) \csc \left(\frac{\pi y}{2}\right) \sec \left(\frac{\pi y}{2}\right)= \\
= & \pi \log \left(\frac{1}{y}\right) \csc ^{2}(\pi y)(\sin (\pi y)-\pi y \cos (\pi y)) \leq 0,
\end{aligned}
$$


because $x \leq \tan x$ for $x \in(0,1)$. Hence $h$ is increasing and tends to $\log (\pi / 2)$ when $y$ tends to zero and this implies the proof.

\section{Lemma 17.}

1) The function

$$
H(y)=\frac{1}{2} \pi \log \left(\frac{1}{y^{y}}\right) \cot \left(\frac{\pi y}{2}\right)-\log \left(\csc \left(\frac{\pi y}{2}\right)\right)
$$

is decreasing from $(0,1)$ onto $(0, \log (\pi / 2))$.

2) The function

$$
G(y)=\log \left(\cosh \left(\frac{\pi y}{2}\right)\right)-\frac{1}{2} \pi y \log (y) \tanh \left(\frac{\pi y}{2}\right)
$$

is increasing from $(0,1)$ onto $(0, \pi \log (\cosh (\pi / 2)) / 2)$.

We get

$$
\begin{aligned}
H^{\prime}(y) & =-\frac{\pi}{4} \csc ^{2}\left(\frac{\pi y}{2}\right)\left(\pi \log \left(y^{-y}\right)+\log (y) \sin (\pi y)\right)= \\
& =-\frac{\pi}{4} \csc ^{2}\left(\frac{\pi y}{2}\right)(\pi y \log (1 / y)-\sin (\pi y) \log (1 / y)),
\end{aligned}
$$

which is positive. Next,

$$
G^{\prime}(y)=-\frac{1}{2} \pi \log (y) \tanh \left(\frac{\pi y}{2}\right)-\frac{1}{4} \pi^{2} y \log (y) \operatorname{sech}^{2}\left(\frac{\pi y}{2}\right)>0,
$$

and the limiting values follow easily.

Lemma 18. The following function is increasing from $(0,1)$ onto $(0, \pi(\log (\pi / 2)) / 2)$

$$
g(x)=\frac{x}{\sqrt{1-x^{2}}} \log \left(\frac{1}{x}\right)-\arcsin (x) \log \left(\frac{1}{\arcsin (x)}\right) .
$$

In particular,

$$
x^{x / \sqrt{1-x^{2}}}<\arcsin (x)^{\arcsin (x)}<\left(\frac{\pi}{2}\right)^{\pi / 2} x^{x / \sqrt{1-x^{2}}} .
$$


We get

$$
\begin{aligned}
g^{\prime}(x) & =-\frac{x^{2} \log (x)}{\left(1-x^{2}\right)^{3 / 2}}-\frac{\log (x)}{\sqrt{1-x^{2}}}+\frac{\log (\arcsin (x))}{\sqrt{1-x^{2}}}= \\
& =\frac{\log (1 / x)-\left(1-x^{2}\right) \log (1 / \arcsin (x))}{\left(1-x^{2}\right)^{3 / 2}}= \\
& =\frac{\log \left(\arcsin (x)^{\left(1-x^{2}\right)} / x\right)}{\left(1-x^{2}\right)^{3 / 2}},
\end{aligned}
$$

which is clearly positive, and $g$ tends to zero when $x$ tends to zero and 1 .

Lemma 19. For $x \in(0,1)$, the following functions

$$
f(k)=\sin \left(\frac{\pi}{2} x^{k}\right)^{1 / k}, g(k)=\tan \left(\frac{\pi}{2} x^{k}\right)^{1 / k}, h(k)=\sinh \left(x^{k}\right)^{1 / k},
$$

are decreasing in $(0, \infty)$. In particular, for $k \geq 1$

$$
\begin{aligned}
\sqrt[k]{\sin \left(\frac{\pi}{2} x^{k}\right)} & \leq \sin \left(\frac{\pi}{2} x\right) \leq \sin \left(\frac{\pi}{2} \sqrt[k]{x}\right)^{k} \\
\sqrt[k]{\tan \left(\frac{\pi}{2} x^{k}\right)} & \leq \tan \left(\frac{\pi}{2} x\right) \leq \tan \left(\frac{\pi}{2} \sqrt[k]{x}\right)^{k}, \\
\sqrt[k]{\sinh \left(x^{k}\right)} & \leq \sinh (x) \leq \sinh (\sqrt[k]{x})^{k}
\end{aligned}
$$

We get

$$
\begin{aligned}
f^{\prime}(k)=\sqrt[k]{\sin \left(\frac{\pi x^{k}}{2}\right)}\left(\frac{\pi x^{k} \log (x) \cot \left(\frac{\pi x^{k}}{2}\right)}{2 k}-\frac{\log \left(\sin \left(\frac{\pi x^{k}}{2}\right)\right)}{k^{2}}\right)= \\
=-\frac{1}{2 k^{2}} \sqrt[k]{\sin \left(\frac{\pi x^{k}}{2}\right)} \times \\
\times\left(\pi k x^{k} \log (1 / x) \cot \left(\frac{\pi x^{k}}{2}\right)-2 \log \left(1 / \sin \left(\frac{\pi x^{k}}{2}\right)\right)\right),
\end{aligned}
$$

which is negative by Lemma 17(1). Next, we get

$$
g^{\prime}(k)=\sqrt[k]{\tan \left(\frac{\pi x^{k}}{2}\right)} \times
$$




$$
\times\left(\frac{\pi x^{k} \log (x) \csc \left(\frac{\pi x^{k}}{2}\right) \sec \left(\frac{\pi x^{k}}{2}\right)}{2 k}-\frac{\log \left(\tan \left(\frac{\pi x^{k}}{2}\right)\right)}{k^{2}}\right) \leq 0,
$$

by (6). Finally,

$$
\begin{aligned}
& h^{\prime}(k)=\sqrt[k]{\sinh \left(x^{k}\right)}\left(\frac{x^{k} \log (x) \operatorname{coth}\left(x^{k}\right)}{k}-\frac{\log \left(\sinh \left(x^{k}\right)\right)}{k^{2}}\right)= \\
& =\sqrt[k]{\sinh \left(x^{k}\right)}\left(x^{k} \log \left(x^{k}\right) \operatorname{coth}\left(x^{k}\right)-\log \left(\sinh \left(x^{k}\right)\right)\right)\left(1 / k^{2}\right)
\end{aligned}
$$

which is negative by inequality (5), and this completes the proof.

Lemma 20. The following functions

$$
\begin{gathered}
f(k)=\cos \left(\frac{\pi}{2} x^{1 / k}\right)^{k}, x \in(0,1), \\
g(k)=\cosh \left(x^{k}\right)^{1 / k}, x \in(0,1), \\
h(k)=\operatorname{arcosh}\left(\frac{\pi}{2} x^{k}\right)^{1 / k}, x \in(1, \infty),
\end{gathered}
$$

are decreasing in $(0, \infty)$. In particular, for $k \geq 1$

$$
\begin{aligned}
\cos \left(\frac{\pi}{2} \sqrt[k]{x}\right)^{k} & \leq \cos \left(\frac{\pi}{2} x\right) \leq \sqrt[k]{\cos \left(\frac{\pi}{2} x^{k}\right)} \\
\sqrt[k]{\cosh \left(x^{k}\right)} & \leq \cosh (x) \leq \cosh (\sqrt[k]{x})^{k}, \\
\sqrt[k]{\operatorname{arcosh}\left(\frac{\pi}{2} x^{k}\right)} & \leq \operatorname{arcosh}\left(\frac{\pi}{2} x\right) \leq \operatorname{arcosh}\left(\frac{\pi}{2} \sqrt[k]{x}\right)^{k} .
\end{aligned}
$$

We get

$$
\begin{gathered}
f^{\prime}(x)=\cos ^{k}\left(\frac{1}{2} \pi x^{1 / k}\right) \times \\
\times\left(\frac{\pi x^{1 / k} \log (x) \tan \left(\pi x^{1 / k} / 2\right)}{2 k}+\log \left(\cos \left(\pi x^{1 / k} / 2\right)\right)\right) \leq 0
\end{gathered}
$$

and proof of $g$ follows from Lemma 14(1). Finally, for $y \geq \pi / 2$, let

$$
j(y)=\operatorname{arcosh}(y) \log (\operatorname{arcosh}(y))-\frac{y \log (2 y / \pi)}{\sqrt{y^{2}-1}},
$$


and

$$
j^{\prime}(y)=\frac{\log (2 y / \pi)}{\left(y^{2}-1\right)^{3 / 2}}+\frac{\log (\operatorname{arcosh}(y))}{\sqrt{y^{2}-1}}>0
$$

and

$$
j(\pi / 2)=\operatorname{arcosh}(\pi / 2) \log (\operatorname{arcosh}(\pi / 2)) \equiv 0.0235 .
$$

With $z=x^{k}$ we get

$$
\begin{gathered}
h^{\prime}(x)=\frac{\operatorname{arcosh}(\pi z / 2)^{1 / k}}{k^{2} \operatorname{arcosh}(\pi z / 2)} \times \\
\times\left(\frac{\pi z \log (z)}{2 \sqrt{(\pi z / 2)^{2}-1}}-\operatorname{arcosh}\left(\frac{\pi}{2} z / 2\right) \log \left(\operatorname{arcosh}\left(\frac{\pi}{2}\right)\right)\right) .
\end{gathered}
$$

This is negative, because $j(y)>0$ for $y>\pi / 2$.

Proof of Theorem 3. The proof for arcosh follows from Lemma 20, and the rest of proof follows from the Lemma 3 , if we take $p=2$.

Lemma 21. The following relations hold

1) $\sin (r) \sin (s)<\sqrt{\sin \left(r^{2}\right) \sin \left(s^{2}\right)}, r, s \in(0,1)$,

2) $\cos (r) \cos (s)<\sqrt{\cos \left(r^{2}\right) \cos \left(s^{2}\right)}<\cos (r s)$,

3) $\tan (r) \tan (s)>\sqrt{\tan \left(r^{2}\right) \tan \left(s^{2}\right)}>\tan (r s)$,

the first inequalities in (2) and (3) hold for $r, s \in(0, \sqrt{\pi / 2})$, and second ones for $r, s \in(0,1)$.

Clearly (1) and the fist inequality of (2) follwos from Lemmas 13 and 20 , respectively. Let $g(x)=\log \left(\cos \left(\pi e^{-x} / 2\right)\right), x>0$, we get

$$
\begin{aligned}
g^{\prime \prime}(x) & =-\frac{\pi^{2}}{4} e^{-2 x} \sec ^{2}\left(\frac{e^{-x} \pi}{2}\right)-\frac{\pi}{2} e^{-x} \tan \left(\frac{e^{-x} \pi}{2}\right)= \\
& =-\frac{\pi}{4} e^{-2 x} \sec ^{2}\left(\frac{e^{-x} \pi}{2}\right)\left(e^{x} \sin \left(e^{-x} \pi\right)+\pi\right) \leq 0,
\end{aligned}
$$

and the second inequality of (2) follows.

For $(3)$, we define $h(x)=\log \left(\tan \left(\pi e^{-x} / 2\right)\right), x>0$, and we get

$$
h^{\prime \prime}(x)=e^{-x} \pi\left(1-e^{-x} \pi \cot \left(e^{-x} \pi\right)\right) \csc \left(e^{-x} \pi\right) \geq 0,
$$

hence $h$ is convex, and the second inequality follows easily, and the first one follows from Lemma 19. 
Lemma 22. For a fixed $x \in(0,1)$, the function $g(k)=(\cos k x+$ $+\sin k x)^{1 / k}$ is decreasing in $(0,1)$.

Differentiation yields

$$
\begin{gathered}
g^{\prime}(k)=(\sin (k x)+\cos (k x))^{\frac{1}{k}} \times \\
\times\left(\frac{k x(\cos (k x)-\sin (k x))}{\sin (k x)+\cos (k x)}-\log (\sin (k x)+\cos (k x))\right) / k^{2} .
\end{gathered}
$$

To prove that this is positive, we let $z=k x, y=\cos z+\sin z \leq 1.1442$

$$
h(z)=(\cos z+\sin z) \log (\cos z+\sin z)-z(\cos z-\sin z),
$$

and observe that

$$
\begin{aligned}
h^{\prime}(z) & =z \cos z+(\cos z-\sin z) \log (\cos z+\sin z)+z \sin z= \\
& =z y+\log y^{\cos z}-\log y^{\sin z} \geq 0,
\end{aligned}
$$

because $e^{z y}>y^{\sin z}$. This implies that $g^{\prime}(k) \geq 0$.

\section{$\S 5$. Appendix}

In the following tables we give the values of $p$-analogue functions for some specific values of its domain with $p=3$ computed with Mathemati$\mathrm{ca}^{\circledR}$. For instance, we can define $[11]$

$\operatorname{arcsinp}\left[\mathrm{p}_{-}, \mathrm{x}_{-}\right]:=\mathrm{x} *$ Hypergeometric2F $1\left[1 / \mathrm{p}, 1 / \mathrm{p}, 1+1 / \mathrm{p}, \mathrm{x}^{\wedge} \mathrm{p}\right]$ $\operatorname{sinp}\left[\mathrm{p}_{-}, \mathrm{y}_{-}\right]:=\mathrm{x} / . \mathrm{FindRoot}[\operatorname{arcsinp}[\mathrm{p}, \mathrm{x}]==\mathrm{y},\{\mathrm{x}, 0.5\}]$

\begin{tabular}{|c|c|c|c|c|c|}
\hline$x$ & $\arcsin _{\mathrm{p}}(x)$ & $\arccos _{\mathrm{p}}(x)$ & $\arctan _{\mathrm{p}}(x)$ & $\operatorname{arsinh}_{\mathrm{p}}(x)$ & $\operatorname{artanh}_{\mathrm{p}}(x)$ \\
\hline 0.00000 & 0.00000 & 1.20920 & 0.00000 & 0.00000 & 0.00000 \\
0.25000 & 0.25033 & 1.17782 & 0.24903 & 0.24968 & 0.25099 \\
0.50000 & 0.50547 & 1.07974 & 0.48540 & 0.49502 & 0.51685 \\
0.75000 & 0.78196 & 0.88660 & 0.68570 & 0.72710 & 0.85661 \\
1.00000 & 1.20920 & 0.00000 & 0.83565 & 0.93771 & $\infty$ \\
\hline
\end{tabular}

\begin{tabular}{|c|c|c|c|c|c|}
\hline$x$ & $\sin _{\mathrm{p}}(x)$ & $\cos _{\mathrm{p}}(x)$ & $\tan _{\mathrm{p}}(x)$ & $\sinh _{\mathrm{p}}(x)$ & $\tanh _{\mathrm{p}}(x)$ \\
\hline 0.00000 & 0.00000 & 1.00000 & 0.00000 & 0.00000 & 0.00000 \\
0.25000 & 0.24967 & 0.99478 & 0.25098 & 0.25033 & 0.24903 \\
0.50000 & 0.49476 & 0.95788 & 0.51652 & 0.50518 & 0.48517 \\
0.75000 & 0.72304 & 0.85362 & 0.84704 & 0.77588 & 0.68283 \\
1.00000 & 0.91139 & 0.62399 & 1.46058 & 1.08009 & 0.82304 \\
\hline
\end{tabular}


B. A. Bhayo, M. Vuorinen

With a normalization different from ours, some eigenvalue problems of the $p$-Laplacian have been studied in [20].

\section{References}

[1] Lindqvist P. Some remarkable sine and cosine functions // Ricerche di Matematica. 1995. Vol. 44. P. 269-290.

[2] Lindqvist P., Peetre J. p-arclength of the $q$-circle // The Mathematics Student. 2003. Vol. 72. P. 139-145.

[3] Biezuner R. J., Ercole G., Martins E. M. Computing the first eigenvalue of the p-Laplacian via the inverse power method // J. Funct. Anal. 2009. Vol. 257, No. 1. P. 243-270.

[4] Biezuner R. J., Ercole G., Martins E. M. Computing the $\sin _{p}$ function via the inverse power method // Comput. Methods Appl. Math. 2011. Vol. 11. No. 2. P. 129-140. arXiv: 1011.3486[math.CA].

[5] Drábek P., Manásevich R. On the closed solution to some p-Laplacian nonhomogeneous eigenvalue problems // Diff. and Int. Eqns. 1999. Vol. 12. P. 723-740.

[6] Reichel W., Walter W. Sturm-Liouville type problems for the p-Laplacian under asymptotic non-resonance conditions // J. Differential Equations. 1999. Vol. 156. No. 1. P. 50-70.

[7] Pino M. del, Elgueta M., Manasevich R. A homotopic deformation along $p$ of a Leray-Schauder degree result and existence for $\left(\left|u^{\prime}\right|^{p-2} u^{\prime}\right)^{\prime}+f(t, u)=0 ; u(0)=u(T)=0 ; p>1 / /$ J. Differential Equations. 1989. Vol. 80. P. 1-13.

[8] Abramowitz M., Stegun I. Handbook of mathematical functions with formulas, graphs and mathematical tables / United states department of commerce. Washington: National Bureau Of Standards, 1964.

[9] Bushell P. J., Edmunds D. E. Remarks on generalised trigonometric functions // Rocky Mountain J. Math. 2012. Vol. 42. P. 25-57.

[10] Takeuchi S. Generalized Jacobian elliptic functions and their application to bifurcation problems associated with p-Laplacian // J. Math. Anal. Appl. 2012 Vol. 385. No. 1. P. 243-255.

[11] Ruskeepää H. Mathematica ${ }^{\circledR}$ Navigator. 3rd ed. [S. 1.]: Academic Press, 2009. 
[12] Neuman E. Inequalities involving inverse circular and inverse hyperbolic functions // Univ. Beograd. Publ. Elektrotehn. Fak. Ser. Mat. 2006. Vol. 18. P. 32-37.

[13] Neuman E. Inequalities involving a logarithmically convex function and their applications to special functions // JIPAM. J. Inequal. Pure Appl. Math. 2006. Article 16.

[14] Anderson G. D., Qiu S.-L., Vamanamurthy M. K., Vuorinen M. Generalized elliptic integrals and modular equation // Pacific J. Math. 2000. Vol. 192, No. 1, P. 1-37.

[15] Anderson G. D., Vamanamurthy M. K., Vuorinen M. Conformal invariants, inequalities and quasiconformal maps. Canadian Mathematical Society Series of Monographs and Advanced Texts, John Wiley \& Sons Inc., New York, 1997.

[16] Anderson G. D., Vamanamurthy M. K., Vuorinen M. Generalized convexity and inequalities // J. Math. Anal. Appl. 2007. Vol. 335. P. 1294-1308.

[17] Kuczma M. An introduction to the theory of functional equations and inequalities. Cauchy's equation and Jensen's inequality / Uniwersytet Slaski. Warszawa; Krakow; Katowice, 1985.

[18] Zhu L. New inequalities of Shafer-Fink Type for arc hyperbolic sine // J. Inequal. Appl. 2008. Art. ID 368275. 5 p. DOI: $10.1155 / 2008 / 368275$.

[19] Klén R., Visuri M., Vuorinen M. On Jordan type inequalities for hyperbolic functions // J. Inequal. Appl. 2010. Art. ID 362548. 14 p. DOI: $10.1155 / 2010 / 362548$.

[20] Brown B. M., Reichel W. Eigenvalues of the radially symmetric $p$-Laplacian in $\mathbb{R}^{n} / /$ J. London Math. Soc. 2004 Vol. 69. No. 3. P. 657-675. DOI: 10.1112/S002461070300512X.

The work is received on May 1, 2013.

University of Turku,

Department of Mathematics and Statistics,

FI-20014 Finland.

E-mail: bhayo.barkat@gmail.com, vuorinen@utu.fi 